\title{
Antihypertensive Therapy Reduces Increased Plasma Levels of Adrenomedullin and Brain Natriuretic Peptide Concomitant with Regression of Left Ventricular Hypertrophy in a Patient with Malignant Hypertension
}

\author{
Toshio Nishikimi, Hiroaki Matsuoka, Kazuhiko Ishikawa, Fumiki Yoshihara, \\ Yuhei Kawano, Kazuo Kitamura*, Yoshihiko Saito**, Kenji Kangawa**, \\ Hisayuki Matsuo**, and Teruo Omae
}

\begin{abstract}
We investigated the potential role of increased plasma adrenomedullin and brain natriuretic peptide (BNP) levels in a patient with malignant hypertension. A 51-year-old man was admitted to our hospital with a chief complaint of visual disturbance. His blood pressure was $270 / 160 \mathrm{mmHg}$ on admission. Papillary edema associated with retinal bleeding was observed. Echocardiography revealed marked concentric left ventricular hypertrophy with mild systolic dysfunction. Plasma levels of adrenomedullin and BNP were markedly elevated. Antihypertensive therapy reduced the plasma levels of adrenomedullin in association with a concomitant decrease in blood pressure. The plasma level of BNP also decreased and regression of left ventricular hypertrophy and normalization of left ventricular systolic function were observed. Our findings suggest that adrenomedullin may be involved in the defense mechanism against further elevations in blood pressure in patients with hypertension and that the plasma level of BNP may reflect left ventricular systolic dysfunction, left ventricular hypertrophy, or both, in patients with severe hypertension. (Hypertens Res 1996; 19: 97-101)
\end{abstract}

Key Words: adrenomedullin, malignant hypertension, brain natriuretic peptide, left ventricular hypertrophy, blood pressure

Adrenomedullin is a novel hypotensive peptide that was discovered in human pheochromocytoma by monitoring of cAMP activity in rat platelets (1). Adrenomedullin consists of 52 amino acids and has one intramolecular disulfide bond. Intravenous administration of adrenomedullin elicits a long-lasting and strong hypotensive effect in rats (1). A significant level of immunoreactive adrenomedullin has been detected in human plasma by a specific radioimmunoassay and high levels of human adrenomedullin mRNA have been demonstrated not only in pheochromocytoma, but also in the normal adrenal gland, the kidney, the lung, the heart, and the vascular walls $(2,3)$. A recent study has shown that vascular smooth muscle cells possess specific adrenomedullin receptors that are functionally coupled to adenylate cyclase (4). In addition, we re- cently found that plasma levels of adrenomedullin are increased in patients with hypertension, renal failure, and heart failure $(5,6)$. These findings indicate that adrenomedullin may be involved in the regulation of the cardiovascular system. In the present study, we measured plasma levels of adrenomedullin using a specific radioimmunoassay (7) before and after antihypertensive treatment in a patient with malignant hypertension associated with left ventricular hypertrophy (LVH) to determine whether adrenomedullin plays a role in this disorder. In addition, we measured the level of brain natriuretic peptide (BNP) using a specific immunoradiometric assay kit with Shiono RIA BNP (Shionogi Co., Ltd., Osaka, Japan) to investigate the relation between LVH or left ventricular systolic function and BNP.

From the Division of Hypertension and Nephrology, National Cardiovascular Center Suita, the *1st Department of Internal Medicine, Miyazaki Medical College, Miyazaki, and ** Research Institute, National Cardiovascular Center, Suita, Japan.

This work was supported in part by Special Coordination Funds for Promoting Science and Technology (Encouragement System of COE) from the Science and Technology Agency of Japan, the Ministry of Health and Welfare, the Human Science Foundation of Japan, and the Ueda Memorial Heart Foundation.

Address for Reprints: Toshio Nishikimi, M.D., Division of Hypertension and Nephrology, National Cardiovascular Center, 5-7-1, Fujishirodai, Suita 565, Japan.

Received September 6, 1995; accepted in revised form November 7, 1995. 


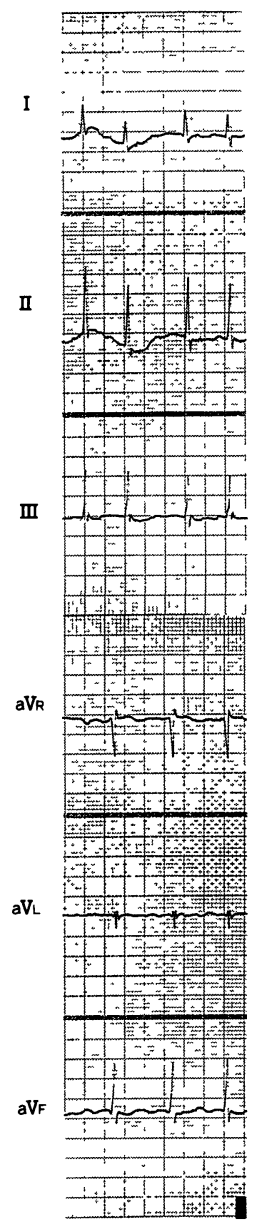

on admission

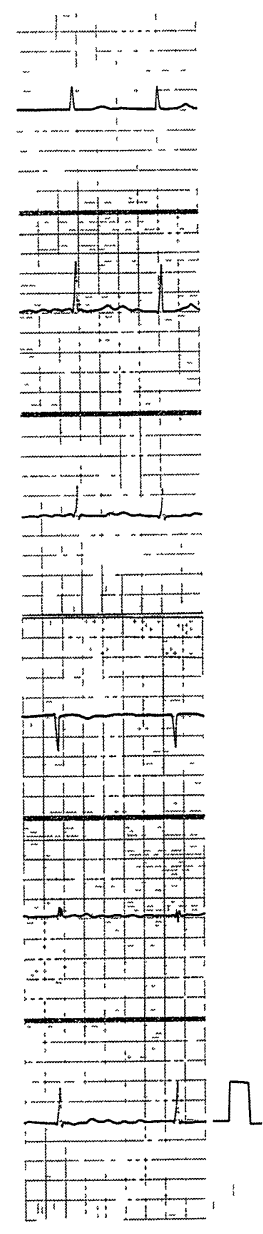

1 month after

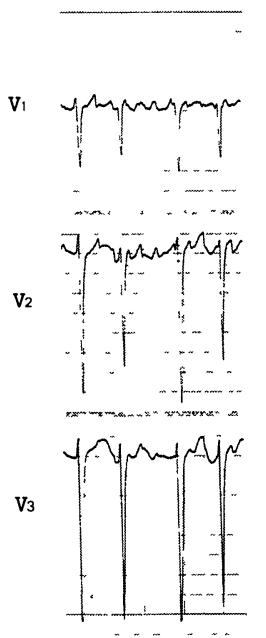

in
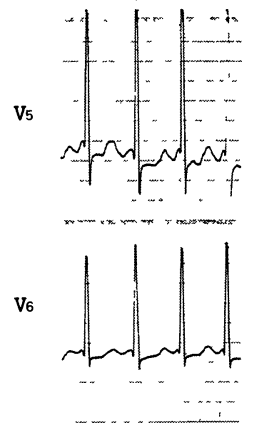

on admission

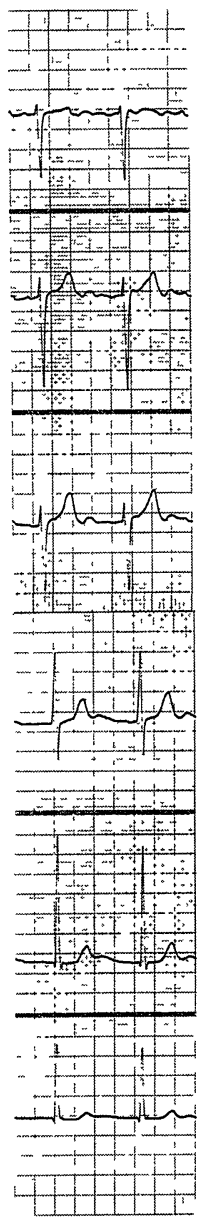

1 month after

Fig. 1. Electrocardiogram before and one month after treatment.

\section{Case Report}

A 51-year-old Japanese man was admitted to our hospital because of visual disturbance. He had a 7year history of atrial fibrillation and a 2-year history of hypertension, but he refused treatment for these conditions. He was not receiving any medication at the time of admission. His blood pressure was $270 / 160 \mathrm{mmHg}$, and he had an irregular pulse of 90 beats/min. His lungs were clear. A grade II/VI pansystolic apical murmur was detected. The findings of abdominal examination were unremarkable. No edema was present. A fundoscopic examination revealed papillary edema with retinal hemorrhage. Laboratory findings on admission were follows: blood urea nitrogen, $23 \mathrm{mg} / \mathrm{dl}$; creatinine, 1.4 $\mathrm{mg} / \mathrm{dl}$; and potassium, $2.9 \mathrm{mmol} / \mathrm{l}$. His white blood cell count was $11.8 \times 10^{9} / 1$, with an increased neutrophil count of $75 \%$. His hemoglobin was 14.3 $\mathrm{gm} / \mathrm{dl}$ and the platelet count was $25.7 \times 10^{10} / 1$. Plasma renin activity (PRA) and plasma levels of aldosterone, norepinephrine, atrial natriuretic peptide (ANP), BNP, and adrenomedullin were increased
(Table 1). Plasma levels of adrenomedullin are 2.5 $\pm 0.2 \mathrm{pmol} / 1$ in our age-matched control $(n=27)$, $2.9 \pm 0.2 \mathrm{pmol} / \mathrm{l}$ in essential hypertension WHO stage I $(n=19)$, and $3.4 \pm 0.3 \mathrm{pmol} / \mathrm{l}$ in essential hypertension WHO stage II $(n=16)$. Plasma levels of BNP are $8 \pm 1 \mathrm{ng} / \mathrm{l}$ in our age-matched control $(n$ $=27), 17 \pm 2 \mathrm{ng} / \mathrm{l}$ in essential hypertension WHO stage I $(n=45)$, and $37 \pm 5 \mathrm{ng} / \mathrm{l}$ in essential hypertension WHO stage II $(n=37)$.

An electrocardiogram showed atrial fibrillation at a ventricular rate of 90 beats/min with an SV2 of $3.5 \mathrm{mV}$, an RV5 of $5.0 \mathrm{mV}$, and ST-segment depression in leads V5 and V6 (Fig. 1). An echocardiogram revealed a mild decrease in left ventricular function, a percent fractional shortening of $24 \%$, mild mitral regurgitation, and hypertrophy of the interventricular septum of $17 \mathrm{~mm}$ and the posterior wall of $14 \mathrm{~mm}$ (Fig. 2). A brain computed tomogram showed a low-density area in the inferior portion of the midbrain to the pons, suggesting the presence of the edema. Intravenous infusion of nicardipine (calcium antagonist) reduced the patient's blood pressure to $150-170 / 90-105 \mathrm{mmHg}$. Oral enalapril therapy was initiated on day 3 . On day 4 , in- 
Table 1. Plasma Hormonal Measurements and Blood pressure on Admission, Day 10, and 1 Month in a Patient with Malignant Hypertension

\begin{tabular}{lccc}
\hline & On admission & Day 10 & 1 month \\
\hline Plasma adrenomedullin $(\mathrm{pmol} / \mathrm{l})^{*}$ & 4.9 & 3.8 & 3.1 \\
Plasma BNP (ng/l) & 330 & 66 & 50 \\
Plasma ANP (ng/l) & 130 & 52 & 31 \\
Plasma norepinephrine (ng/l) & 740 & 180 & 220 \\
Plasma aldosterone (ng/l) & 360 & 130 & 160 \\
Plasma renin activity (ng/ml/h) & 28.0 & 25.0 & 24.0 \\
SBP/DBP (mmHg) & $270 / 160$ & $166 / 106$ & $154 / 92$ \\
\hline
\end{tabular}

BNP: brain natriuretic peptide, ANP: atrial natriuretic peptide, SBP: systolic blood pressure, DBP: diastolic blood pressure. Note that PRA values on day 10 and 1 month were obtained while the patient was receiving an angiotensin-converting enzyme inhibitor; *age matched controls: $2.5 \pm 0.2(n=27)$.
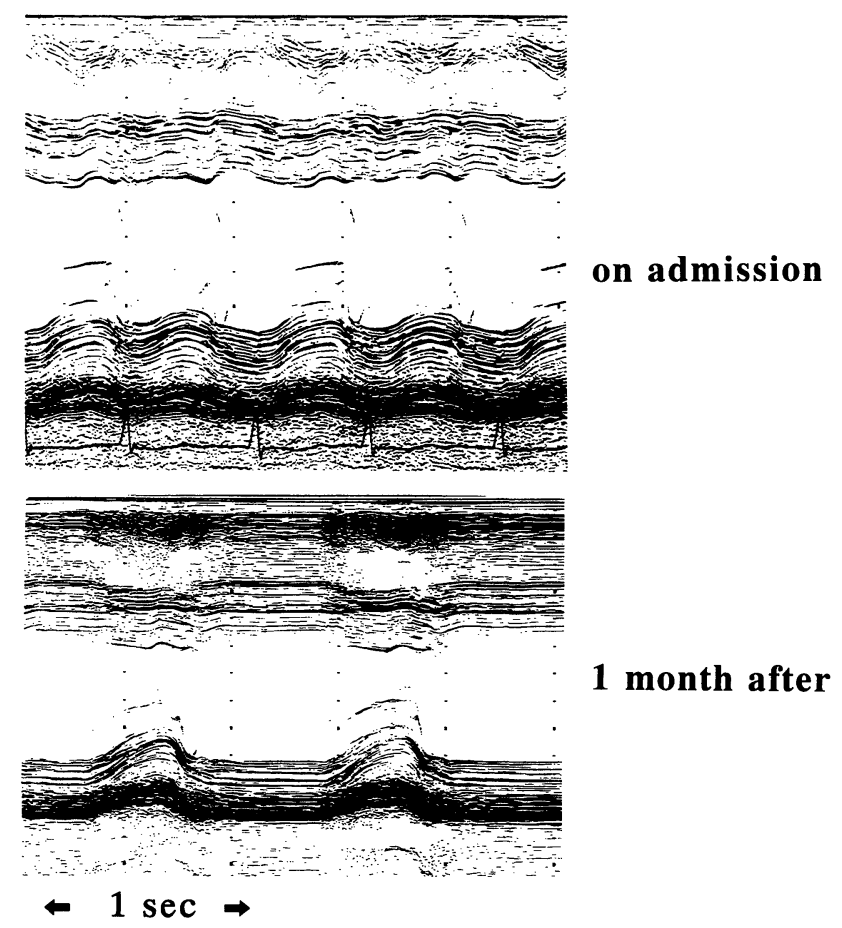

1 month after

Fig. 2. M-mode echocardiogram before and one month after treatment.

travenous nicardipine was replaced by oral nifedipine. On day 10, his blood pressure was 166/106 $\mathrm{mmHg}$ and his heart rate 67 beats/min. The plasma levels of BNP and adrenomedullin decreased markedly (Table 1). An echocardiogram revealed improvement of left ventricular function, with a percent fractional shortening of $30 \%$. The interventricular septal thickness decreased to $14 \mathrm{~mm}$ and the posterior wall thickness to $12 \mathrm{~mm}$. The patient's blood pressure was maintained finally at $140-155 /$ $85-95 \mathrm{mmHg}$ by combination therapy with $80 \mathrm{mg} / \mathrm{d}$ of oral nifedipine and $5 \mathrm{mg} / \mathrm{d}$ of enalapril. One month later, his blood pressure was $154 / 92 \mathrm{mmHg}$ and heart rate 60 beats $/$ min. Plasma levels of BNP and of adrenomedullin showed further decreases (Table 1). The electrocardiogram showed an improvement of LVH, with an SV2 of $2.2 \mathrm{mV}$ and an
RV5 of $3.0 \mathrm{mV}$. No ST-segment depression was observed in leads in V5 and V6 (Fig. 1). An echocardiogram showed further regression of $\mathrm{LVH}$, with an interventricular septal thickness of $12 \mathrm{~mm}$ and a posterior wall thickness of $11 \mathrm{~mm}$ (Fig. 2). However, serum creatinine and blood urea nitrogen levels did not change after the treatment.

\section{Discussion}

This study had two main findings. Firstly, the plasma level of adrenomedullin was increased in our patient with malignant hypertension; antihypertensive therapy reduced the plasma level of adrenomedullin in association with a reduction in blood pressure. Secondly, the plasma level of BNP was also elevated and was reduced by antihypertensive treatments in association with regression of $\mathrm{LVH}$ and normalization of left ventricular systolic function.

We previously reported that the plasma levels of adrenomedullin were increased in proportion to the severity of target organ damage in patients with essential hypertension (5). Findings in the present case appear to confirm our previous findings. The plasma levels of adrenomedullin in the present case were higher than levels in patients with WHO stage II essential hypertension (5). Although the origin of circulating adrenomedullin in humans has not been determined (8), Sugo et al. reported that endothelial cells and vascular smooth muscle cells produce and secrete adrenomedullin in vitro (3). Cytokines, such as interleukin-1 and tumor necrosis factor, and vasoactive substances such as angiotensin II, aldosterone, and norepinephrine, have been found to stimulate adrenomedullin production in vascular smooth muscle cells $(3,9,10)$. Renin-angiotensin aldosterone system and sympathetic nervous system were activated in the present case, suggesting that activation of these neurohumoral factors may have contributed to the increase in the plasma levels of adrenomedullin. However, plasma levels of adrenomedullin have been found to increase in proportion to the severity of renal failure in patients with renal failure (5). The creatinine level was slightly elevated in our patient, suggesting that renal impairment may also have contributed to the increase in the plasma level of adrenomedullin. Interestingly, 
antihypertensive treatment reduced the plasma level of adrenomedullin. Although it is unknown whether an increase in blood pressure itself stimulates the production or secretion of adrenomedullin, adrenomedullin levels in our patient appeared to be related to the blood pressure level. It is possible that adrenomedullin, which is a novel vasorelaxant peptide, may be involved in the defense mechanism against further elevations in blood pressure in patients with hypertension.

In the present study, echocardiography revealed marked concentric LVH associated with mild systolic dysfunction. Shapiro and Beevers have suggested that patients with malignant hypertension who have a previous history of hypertension tend to have $\mathrm{LVH}$, whereas those without a previous history of hypertension tend not to have LVH (11). Our patient had a 2-year history of hypertension. However, Shapiro and Beevers reported that LVH in patients with malignant hypertension did not change, despite an adequate reduction in blood pressure for six months (11), whereas in our patient, antihypertensive therapy was associated with clear regression of LVH within 1 month. Although we cannot explain the discrepancy between our findings and those of Shapiro and Beevers, it is possible that improvements in antihypertensive drug therapy may be responsible. In fact, it was reported that 4-8 weeks' antihypertensive treatment with a angiotensin-converting enzyme inhibitor or calcium antagonist decreased the left ventricular mass in essential hypertensive subjects (12). Our patient's blood pressure was easily controlled by intravenous administration of a calcium antagonist and oral therapy with an angiotensin-converting enzyme inhibitor. Angiotensin-converting enzyme inhibitors cause the greatest degree of regression of LVH in patients with hypertension (13). Neither calcium antagonists nor angiotensin-converting enzyme inhibitors were available at the time of the study by Shapiro and Beevers.

In the present case, the plasma level of BNP was markedly elevated on admission and was reduced by antihypertensive treatment in association with regression of LVH and normalization of left ventricular systolic function. BNP secretion is induced primarily by myocyte stretch in the left ventricle, and the plasma levels of BNP are related to left ventricular function (14). In the present case, blood pressure was markedly elevated and left ventricular function was impaired on admission, suggesting that the left ventricular systolic pressure and end-diastolic pressure may have been markedly elevated, which may have stimulated BNP secretion. Kohno et al. reported that the plasma levels of BNP were elevated in hypertensive subjects with LVH (15). We also found that plasma levels of BNP are increased in subjects with essential hypertension who have concentric LVH, and that BNP levels show a stronger correlation with the interventricular septal thickness and the posterior wall thickness than with blood pressure levels (16). In the present case, a reduction in the plasma level of BNP was associated with regression of LVH. These findings suggest that the plasma levels of BNP may reflect left ventricular function, the degree of $\mathrm{LVH}$, or both, in hypertensive subjects.

To our knowledge, this is the first report to document increased plasma levels of adrenomedullin and BNP in a patient with malignant hypertension. Our findings suggest that adrenomedullin may be involved in the defense mechanism for further increases in blood pressure, and that the plasma level of BNP may be related to LVH, left ventricular systolic dysfunction, or both, in patients with severe hypertension. Delineation of the exact cellular mechanism responsible for the production and secretion of these peptides requires further investigation.

\section{Acknowledgment}

The technical assistance of Ms. Yoko Saito is gratefully acknowledged.

\section{References}

1. Kitamura K, Kangawa K, Kawamoto M, et al: Adrenomedullin: a novel hypotensive peptide isolated from human pheochromocytoma. Biochem Biophys Res Commun 1993; 192: 553-560.

2. Kitamura K, Sakata J, Kangawa K, Kojima M, Matsuo $\mathrm{H}$, Eto T: Cloning and characterization of cDNA encoding a precursor for human adrenomedullin. Biochem Biophys Res Commun 1993; 194: 720-725.

3. Sugo S, Minamino N, Shoji $\mathrm{H}$, et al: Production and secretion of adrenomedullin from vascular smooth muscle cells: augmented production by tumor necrosis factor- $\alpha$. Biochem Biophys Res Commun 1994; 203: 719-726.

4. Eguchi S, Hirata Y, Kano H, et al: Specific receptors for adrenomedullin in cultured rat vascular smooth muscle cells. FEBS Lett 1994; 340: 226-230.

5. Ishimitsu $\mathrm{T}$, Nishikimi $\mathrm{T}$, Saito $\mathrm{Y}$, et al: Plasma levels of adrenomedullin, a hypotensive peptide, in patients with hypertension and renal failure. J Clin Invest 1994; 94: 2158-2161.

6. Nishikimi T, Saito Y, Kitamura K, et al: Increased plasma levels of adrenomedullin in patients with heart failure. J Am Coll Cardiol 1995; 26: 1424-1431.

7. Kitamura $\mathrm{K}$, Ichiki $\mathrm{Y}$, Tanaka $\mathrm{M}$, et al: Immunoreactive adrenomedullin in human plasma. FEBS Lett 1994; 341: 288-290.

8. Nishikimi T, Kitamura K, Saito Y, et al: Clinical studies for the sites of production and clearance of circulating adrenomedullin in human subjects. Hypertension 1994; 24: 600-604.

9. Minamino N, Sugo S, Shoji H, Kangawa K, Matsuo $\mathrm{H}$ : Adrenocortical steroids, thyroid hormones and retinoic acid augment the production of adrenomedullin in vascular smooth muscle cells. Biochem Biophys Res Commun 1995; 211: 686-693.

10. Shoji H, Minamino N, Sugo S, Kangawa K, Matsuo $\mathrm{H}$ : Effects of vasoactive substances and cyclic AMP related compounds on adrenomedullin production in cultured vascular smooth muscle cells. FEBS Lett 1995; 369: 311-314.

11. Shapiro LM, Beevers DG: Malignant hypertension: cardiac structure and function at presentation and during therapy. Br Heart J 1983; 49: 477-484.

12. Aristizabel D, Messerli FH, Frohlich ED: Disparate structural effects on left and right ventricles by 
angiotensin-converting enzyme inhibitors and calcium antagonist in essential hypertension. Am J Cardiol 1994; 73: 483-487.

13. Dahlöf B, Pennert K, Hansson L: Reversal of left ventricular hypertrophy in hypertensive patients: a meta analysis of 109 treatment studies. Am J Hypertens 1992; 5: 95-110.

14. Yasue H, Yoshimura M, Shimada H, et al: Localization and mechanism of secretion of B-type natriuretic peptide in comparison with those of A-type natriure- tic peptide in normal subjects and patients with heart failure. Circulation 1994; 90: 195-203.

15. Kohno $\mathrm{M}$, Horio $\mathrm{T}$, Yokokawa $\mathrm{K}$, et al: Brain natriuretic peptide as a cardiac hormone in essential hypertension. Am J Med 1992; 92: 29-34.

16. Nishikimi T, Yoshihara F, Morimoto A, et al: Relationship between left ventricular geometry and natriuretic peptide levels in essential hypertension. Hypertens 1996 (in press). 